\title{
¿Eutanasia o limitación del esfuerzo terapéutico?
} Euthanasia or limitation of therapeutic effort?

El desarrollo tecnológico, la aplicación de nuevas modalidades terapéuticas y el crecimiento de las unidades de cuidados intensivos pediátricas han permitido recuperar muchos niños con enfermedades antes consideradas mortales. El progreso generó un cambio de paradigma, en el cual, la muerte es una circunstancia siempre prevenible, evitable. En este contexto, la muerte de un niño resulta aún más inaceptable. Sin duda, el lado escabroso de este nuevo paradigma es la prolongación de la agonía y el sufrimiento en aquellos casos en que el tratamiento es fútil, lo cual genera una forma de iatrogenia definida como encarnizamiento terapéutico.

Los cuidados paliativos surgen como una respuesta para mitigar el sufrimiento y sostener al niño y a la familia en tan duro trance. Al definir el cuidado paliativo como "... el cuidado activo y total de las enfermedades que no tienen respuesta al tratamiento curativo, siendo el objetivo principal conseguir la mejor calidad de vida posible para los pacientes y sus familias", la Organización Mundial de la Salud ofrece algunos lineamientos útiles: alivio del dolor y de otros síntomas; no alargar ni acortar la vida; considerar la muerte como algo normal; proporcionar sistemas de apoyo para que la vida sea lo más activa posible y dar apoyo a la familia durante la enfermedad y el duelo. ${ }^{1}$

Frente a un niño gravemente enfermo hay numerosas decisiones por consensuar y se requiere un laborioso trabajo interdisciplinario cuando se trata de definir la licitud de qué hacer o dejar de hacer. Existe una tendencia creciente a utilizar tratamientos intensivos y concentrar recursos para lograr la supervivencia de niños, por ejemplo, con malformaciones gravísimas, que -tras la aplicación de costosos cuidados intensivos, máximos y prolongados- sobreviven con marcadas secuelas, escasísima calidad de vida y, finalmente, enfrentan la muerte a muy temprana edad. ${ }^{2}$

En nuestro país, en algunas ocasiones, fue la Justicia la que decidió cuándo continuar o concluir el soporte pese a que la instancia legal no es la indicada para tomar decisiones que competen a la familia y al personal de salud, quienes, junto con los comités hospitalarios de bioética, son los indicados para arribar a soluciones que den sostén al equipo sanitario y a la familia en la toma de decisiones. ${ }^{3}$
En la Argentina se comenzó a debatir la aprobación de la "eutanasia" con sendas audiencias en el Senado y la Legislatura de Buenos Aires, luego de la conmoción que causó el caso de una pareja que pide una "muerte digna" para su hija de 2 años, en estado vegetativo desde su nacimiento. La discusión sobre la "muerte digna" llegó a las portadas de los diarios con el caso de la pequeña Camila, quien sobrevive conectada a un respirador artificial. ${ }^{4}$

Según la madre "Camila está en estado vegetativo desde que nació. Todos afirman y confirman que el estado de la niña es irreversible". En abril de 2009, Camila "nació muerta, se la reanimó durante 20 minutos y se la conectó a un respirador artificial. Tuvo una encefalopatía crónica a raíz de la falta de oxígeno en el momento del parto", aseguró su madre e instó a los legisladores a reactivar los distintos proyectos de ley que están en estudio en el Parlamento desde hace varios años.

La madre de Camila pide se deje morir a su hija, pero los médicos se niegan a hacerlo porque en la Argentina no existe legislación que lo permita. Afirmó que los comités de bioética de entidades públicas y privadas han respaldado su petición de desconectar a Camila: "La situación por la que estamos pasando como familia es dolorosa, considerando que tenemos una beba en estado vegetativo permanente. La situación de mi hija es irrecuperable e irreversible, y hay un vacío legal que impide retirar el soporte vital". Estas líneas son parte de una carta que la mujer ha enviado a la Cámara de Diputados argentina, en la cual explica la situación en la que se encuentra la niña: "No oye, no ve, no llora, no parpadea, no traga, no se mueve, no siente nada y desde que nació está conectada a un respirador artificial" ${ }^{3}$

Los senadores presentaron varios proyectos que abordan la modificación de los derechos del paciente, el consentimiento informado, la protección de la calidad de vida frente a diagnósticos médicos y la incorporación del Registro de Voluntad Anticipada para que cualquier persona, en buenas condiciones de salud, pueda consignar su decisión ante una eventual situación límite. "Se trata de que haya una respuesta del Estado a través de una ley que facilite ese trance tan duro. Cada vez hay más médicos que se dan cuenta de la limitación terapéutica cuando ésta se vuelve cruenta y es una combinación de aparatos y far- 
macología", evaluó en declaraciones radiales el senador Samuel Cabanchik, uno de los impulsores de la iniciativa.

La prensa hace referencia a la eutanasia y lo que se discute en el caso Camila es, claramente, la limitación del esfuerzo terapéutico y fue esta la razón que avaló la recomendación efectuada por los comités de bioética intervinientes. Recordemos que las recomendaciones de los comités no son jurídicamente vinculantes, pero no quiere decir que su decisión no tenga peso y está fundada en la ética aplicada a la salud. Beatriz Firmenich hace hincapié en que "la recomendación del comité pide la limitación del esfuerzo terapéutico, algo que sí está contemplado en el marco jurídico argentino. La limitación del esfuerzo terapéutico acontece cotidianamente, lo que sucede es que, en este caso, la madre de Camila se ha visto movida a llevarlo a los medios de comunicación porque ha pasado por tres comités de ética y, aun así, su demanda no ha sido escuchada en el hospital". Tres comités de bioética argentinos, tanto públicos como privados, han aconsejado que se escuche la petición de la madre, pero, en el hospital donde está ingresada Camila, las autoridades argumentan que no pueden practicar la eutanasia porque no existe legislación que los avale y aquí cometen el mismo error: limitación de soporte vital no es eutanasia. La madre de Camila está dispuesta a llegar a todas las instancias posibles y ha denunciado el caso ante el Ministerio de Justicia y Derechos Humanos de la Nación. ${ }^{5}$

Estos casos deberían ser resueltos de común acuerdo entre la familia del paciente y el equipo de salud. Los profesionales no están obligados a mantener un tratamiento que resulta ineficaz. $Y$ no se trata de poner fin a su vida, acción definida como eutanasia, sino de permitir que la muerte acontezca. Son los Comités de Bioética institucionales los que deben asesorar al equipo médico y a la familia cuando surgen conflictos, creando un marco de confianza, que transmita alivio por estar acompañados en la toma de decisiones. Resta aún una segunda instancia: presentar el caso a un comité de bioética ajeno a la institución, para evitar comprometer a su personal, juez y parte en la deliberación. De hecho, cabe destacar que un juez en lo criminal ha dicho: "La interrupción de medios artificiales de sostén vital en casos de un estado vegetativo persistente, previo dictamen de una junta médica y/o Comité de Etica, en diálogo con familiares directos del paciente, resulta inobjetable". ${ }^{6}$

Mientras tanto, si no hay consenso, si continúa tergiversándose el término eutanasia, los pacientes en estado vegetativo permanente seguirán conectados a las máquinas que les permiten permanecer con "vida".

Dra. Teresa Pereira Silva

Presidenta de la Subcomisión de Bioética SAP

http:/ /dx.doi.org/10.5546/aap.2012.203

1. Levin R. Cuidados paliativos: Principios generales. OMSOPS.[Acceso 7 de octubre de 2011]. Disponible en: http:// www.paho.org/spanish/ad/dpc/nc/palliative-care-01.pdf.

2. Gherardi CR. La muerte intervenida: una visión comprensiva desde la acción sobre el soporte vital. Perspectivas bioéticas 2006;20:102-21.

3. Gherardi CR, Gherardi N. La judicialización del acto médico y la generación de nuevos conflictos. Medicina (B. Aires) 2007; 67(5):502-10.

4. Argentina comienza a debatir leyes sobre "muerte digna". El Nuevo Diario.com 2011 sept 27. [Acceso 1 de octubre de 2011]. Disponible en: http:/ / www.elnuevodiario.com.ni/ internacionales /115118_argentina-comienza-a-debatir-leyes-sobre.

5. Díez Hernando B. Argentina: debate en torno a la muerte digna. Radio Nederland Internacional. 2011 Agos 19. [Acceso 3 de octubre de 2011]. Disponible en: http:/ / www.rnw. $\mathrm{nl} /$ espanol/article/argentina-debate-en-torno-a-la-muertedigna.

6. Blanco, LG. Bioética: cuestiones abiertas. Inicio, desarrollo y fin de la vida humana. Buenos Aires; Eledé, 1996.

\section{Por qué NO usar la palabra accidente}

\section{Why not use the word accident}

La palabra "accidente" está muy extendida en el ámbito social y en los medios masivos de comunicación, y resulta difícil reemplazarla. El término significa literalmente "lesiones provocadas sin intencionalidad que ocasionan daños a las personas y que ocurren en forma brusca o imprevista".

Las muertes traumáticas a lo largo de la historia se relacionaron con las guerras. Al comenzar la industrialización y la urbanización, durante el siglo XIX, se incrementaron los accidentes. Es 
decir que estos tienen una directa relación con el desarrollo tecnológico. Los accidentes laborales durante el siglo antepasado eran interpretados como un costo inevitable de la producción, una contingencia de la vida. La idea predominante hasta mediados del siglo XX era que los accidentes constituían una consecuencia no deseada, pero inevitable, del progreso. Culturalmente ese concepto se trasladó hasta bastante avanzado el siglo XX, y de esta concepción proviene el considerarlos como "actos de Dios" o cosas del destino o la fatalidad. ${ }^{2}$

Por eso es que la palabra accidente, que debiéramos nombrar de otra manera, carga un significado de inevitabilidad que tiene implicancias: en efecto, si son inevitables, nada podemos hacer para prevenirlos.

En ámbitos de salud pública y académicos existe desde hace varios años una tendencia creciente a reemplazarla, pues las connotaciones de la palabra no reflejan lo que se quiere expresar. Al igual que en otras áreas, en el ámbito de la salud y la medicina es muy importante usar los términos con precisión. El uso de un lenguaje claro apoya la comprensión de la real significación de los eventos, contribuye a su prevención y control, y facilita la comunicación en el campo científico. ${ }^{3}$

Sin embargo, el reemplazo de un término tan arraigado no es sencillo, ni desde el punto de vista de su acepción cultural ni desde la lingüística. Organismos y publicaciones anglosajonas en general utilizan el término "injury", que podríamos traducir como "lesiones". Muchos autores utilizan también la palabra trauma.

Pero una lesión es la consecuencia de un evento, y no habla de su mecanismo de producción. Las causas externas de morbilidad y mortalidad, codificadas en la Clasificación Internacional de Enfermedades (CIE 10) ${ }^{4}$ clasifican a los eventos externos que producen daños según su intencionalidad: no intencionales (mal llamados accidentes), e intencionales: agresiones y suicidios.

Las lesiones no intencionales devienen de la exposición a factores externos, tales como niveles intolerables de energía (mecánica, radiante, eléctrica, química), traumatismos en los ámbitos laborales y lesiones por tránsito o viales. ${ }^{5}$

Si bien en la infancia son la causa más frecuente de enfermedad y muerte, las lesiones no intencionales, la violencia y los suicidios cobran una importancia creciente y dramática en nuestra sociedad. En ocasiones, el límite entre lesiones intencionales y no intencionales puede no ser muy claro: negligencia en el cuidado de niños peque- ños, situaciones de abandono, choques causados por conducción peligrosa o sujetos alcoholizados, etc.

William Haddon, considerado el padre de la ciencia de la prevención del trauma, en la década del 60 ideó el principal modelo conceptual con el que se evalúan las causas y se identifican objetivos para el desarrollo de medidas y estrategias de prevención y control. En él distingue claramente entre el individuo, el entorno y el agente productor de la lesión resultante, por un lado; por otro incorpora el factor tiempo describiendo tres fases: pre evento, evento y post evento. Se crea así una matriz de 9 celdas que permite definir gráficamente los eventos, situaciones o acciones que incrementan o disminuyen la probabilidad de que un trauma ocurra, a la vez que permite diseñar estrategias de prevención. Además, demuestra que múltiples factores pueden llevar a producir lesiones y que existen muchas oportunidades para prevenirlas o para reducir su gravedad. Ya entonces Haddon expresaba que los conceptos de suerte, azar o accidente eran precientíficos, y que sobrevivían de épocas oscuras en las que el hombre desconocía las fuerzas que causaban enfermedades, plagas y terremotos y para las cuales no tenía una explicación racional. ${ }^{6}$

Si bien la palabra accidente no cubre adecuadamente la complejidad del fenómeno y añade un supuesto etiológico erróneo, las palabras lesión o trauma solo representan el resultado de un proceso en el cual un hecho, anteriormente conocido como accidente, desempeña un papel primordial. ${ }^{3}$

Organismos internacionales de prestigio han dejado de utilizar el término "accidente" para referirse a las lesiones no intencionales. La National Highway Traffic Safety Administration (NHTSA) es una agencia del gobierno de los Estados Unidos, que desde 1996 se propone reducir la cantidad de víctimas fatales en el tránsito mediante el uso universal de cinturones de seguridad. Su campaña se llamó "Crashes aren't accidents", (los choques no son accidentes). ${ }^{7}$ Una de las secciones más relevantes de la Academia Norteamericana de Pediatría está constituida por el "Consejo sobre prevención de lesiones, violencia e intoxicaciones" (COIVPP). También el Centro para el Control y la Prevención de Enfermedades (CDC), la agencia de salud pública del gobierno de los EE.UU., tiene una amplia sección sobre "Lesiones, Violencia y Seguridad".

Hace ya 10 años, la revista British Medical Journal publicó un editorial cuyo título era: "BMJ bans 'accidents'. Accidents are not unpredictable" (BMJ 
prohíbe 'accidentes'. Los accidentes no son imprevisibles), en el cual expresaba que la mayoría de las lesiones y los eventos que los causan son previsibles y prevenibles, y por eso el BMJ decidió prohibir la palabra accidente en los artículos que publicaba. ${ }^{8}$

Así, la Subcomisión de Prevención de Accidentes de la SAP ha cambiado su denominación por Subcomisión de Prevención de Lesiones, en el convencimiento de que el uso preciso del lenguaje apoya la comprensión de la real significación de los eventos. Las lesiones de todo tipo: en el hogar, en la escuela, en los lugares de recreación, en el tránsito, se pueden prevenir.

Nos gustaría hacer extensivo este criterio a los artículos publicados en Archivos Argentinos de Pediatría: pedir a autores, revisores y lectores reemplazar la palabra accidente por otro término que refleje mejor lo que significan las lesiones traumáticas. Comprendiendo y aplicando estos conceptos, se podrán implementar mejores estrategias de prevención que ayuden a reducir la alta incidencia, y la gravedad de lesiones no intencionales en los niños, adolescentes y jóvenes.
Dra. Ingrid Waisman

Presidente Subcomisión de Prevención de Lesiones Sociedad Argentina de Pediatría

http:/ /dx.doi.org/10.5546/aap.2012.204

1. Glizer IM. Prevención deaccidentes y lesiones. Washington: Serie Paltex. Organización Panamericana de la Salud, 1993.

2. Songer T. History of injury epidemiology. [Acceso: 18 enero 2012]. Disponible en: http://www.pitt.edu/ epi2670/ injuryhistory/History2009.pdf.

3. Ruiz Pérez, M. ¿Se debe usar el término accidente en el ámbito de la investigación científica? Panace@ 2011;12(33):84-8.

4. Organización Panamericana de la Salud. Clasificación estadística internacional de enfermedades y problemas relacionados con la salud. $10^{\mathrm{a}}$ revisión. Washington, DC: OPS; 1995.

5. Zein O, Úbeda C, Vacchino M. Lesiones por causas externas. Boletín Epidemiológico periódico. Buenos Aires: Ministerio de Salud de la Nación, 2007.

6. Haddon W Jr. The changing approach to the epidemiology, prevention, and amelioration of trauma: the transition to approaches etiologically rather than descriptively based. Am J Public Health Nations Health 1968;58(8):1431-8.

7. NHTSA. Initiative for increasing seat belt use nationwide. [Acceso: 17 enero 2012]. Disponible en: http://www. nhtsa.gov/people/injury/airbags/Archive-04/PresBelt/ nations_goals.html.

8. Davis R, Pless B. BMJ bans "accidents". BMJ 2001; 322 (7298):1320-1. 